\title{
THE USE OF ENZYMATIC DEBRIDEMENT IN LARGE BURNS : TWO YEARS OF EXPERIENCE
}

J. MINIC, E. VIGATO, M. GOVERNA

Azienda Ospedaliera Universitaria Integrata Verona

The modern era of treatment of burn injury' started a half century ago, in the early seventies with the revolutionary approach of early excision debridement followed by autologous skin grafting. For many decades it's been the gold standard saving many lives and giving us the insight of the basic requirements the better burn care. Surgery is an effective debridement strategy although not selective, ending in massive losses of not injured, vital tissues (mainly dermis), blood an heat.

We hypothesized that selective enzymatic debridement (ED) could have a positive outcome by reducing blood, heat and dermal losses, offering a better local control of wound healing and decreasing local and systemic response

\section{MATERIAL AND METHODS}

All patients were treated on admission besides resuscitation by cleaning the burns, removing any previous topical medications and the blisters. The clean burn wound were soaked with normal saline until ED application (within $24 \mathrm{~h}$ ). Following removal of the ED dressing the dissolved escaper and remaining ED were wiped away using sterile wooden tongue depressor. A single ED application sufficed remove the entire eschar. The wound was soaked with normal saline at least 2 hours an then final diagnosis was made.The derided bed was covered with allograft that were left in place until spontaneous epithelisation or autographing of non healing wounds.

\section{RESULTS}

\begin{tabular}{|c|c|c|}
\hline & 2017 & 2018 \\
\hline $\mathbf{N}^{\circ}$ PT E.D. (LARGE BURNS) & $|6 / 2|$ & $19 / 29$ \\
\hline $\begin{array}{l}\text { AGE } \\
(18-96 \text { YS) }\end{array}$ & 50,92 & 50,5 \\
\hline $\begin{array}{l}\text { RANGE } \% \text { TBSA } \\
\text { L.B. }>15 \%\end{array}$ & $18 \div 85 \%$ & $15 \div 65 \%$ \\
\hline \%TBSA ADMISSION (MEAN) & $33,29 \%$ & $30,91 \%$ \\
\hline $\begin{array}{l}\text { \% TBSA TERATED } \\
\text { ( MEAN ) }\end{array}$ & $27,5 \%$ & $29,5 \%$ \\
\hline $\begin{array}{l}\text { \% SKIN GRAFTS } \\
\text { ( MEAN) }\end{array}$ & $7 \%$ & $5,1 \%$ \\
\hline $\begin{array}{l}\text { INFECTION } \\
\text { CONTAMINATION VS SEPSIS }\end{array}$ & I 3 VS 4 & I7 VS 2 \\
\hline $\begin{array}{l}\text { BLOOD TRANSFUSION AFTER } \\
\text { E.D. }\end{array}$ & $\mathbf{I}$ & $\mathbf{0}$ \\
\hline $\mathbf{N}^{\circ}$ DAYS OF RECOVERY & 44,2 I & 49,94 \\
\hline $\begin{array}{c}\text { PLACE OF } \\
\text { PERFORMANCE OF } \\
\text { E.D. }\end{array}$ & 2017 & 2018 \\
\hline SICU & 7 & 13 \\
\hline IC & 9 & 14 \\
\hline OR & 7 & 2 \\
\hline
\end{tabular}

PERIOD:

FEB 2017- FEB 2019

$N^{\circ}$ OFTOTAL ED CASES :

$N^{\circ}$ OF ED CASES ABOVE I5\% TBSA:

AGE (MEAN) :

50,7IYS (I8 - 96YS)

GENDER

24M, IIF

AVERAGE OFTBSA \% :

$27,36 \%(15 \%-85 \%)$

TBSA\% \& ED

$28,5 \%$

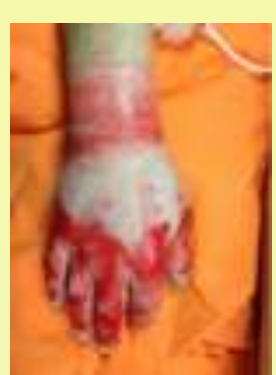

ADMISSION

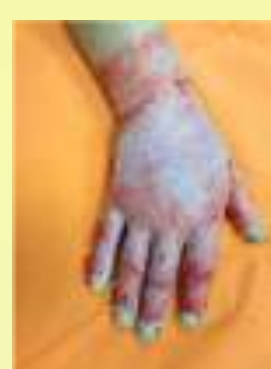

AFTER ED

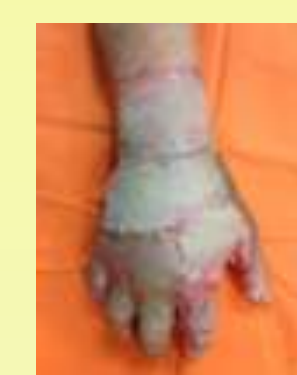

ALLOGRAFTS

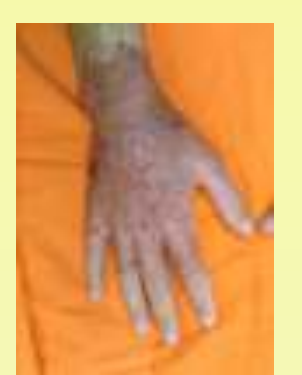

EPITHELISATION

- AREA OF APPLICATION ON THE SINGLE PATIENT: 30\% TBSA

- MAX APPLICATION AREA ON SINGLE PATIENT : 60\% TBSA

- MAX AGE TREATED WITH E.D. 96 YS
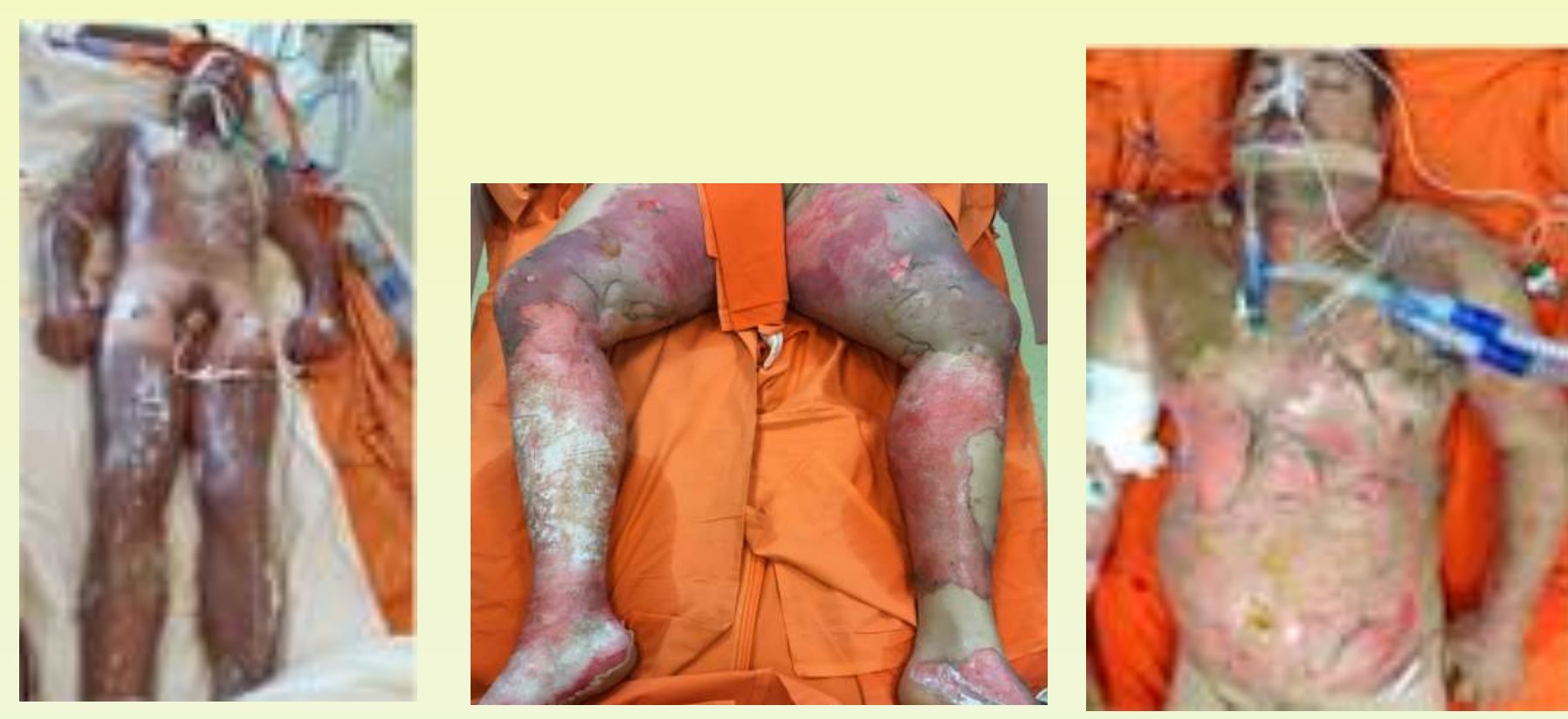

LARGE DEEP FLAME BURNS ON ADMISSION PRIOR TREATMENT
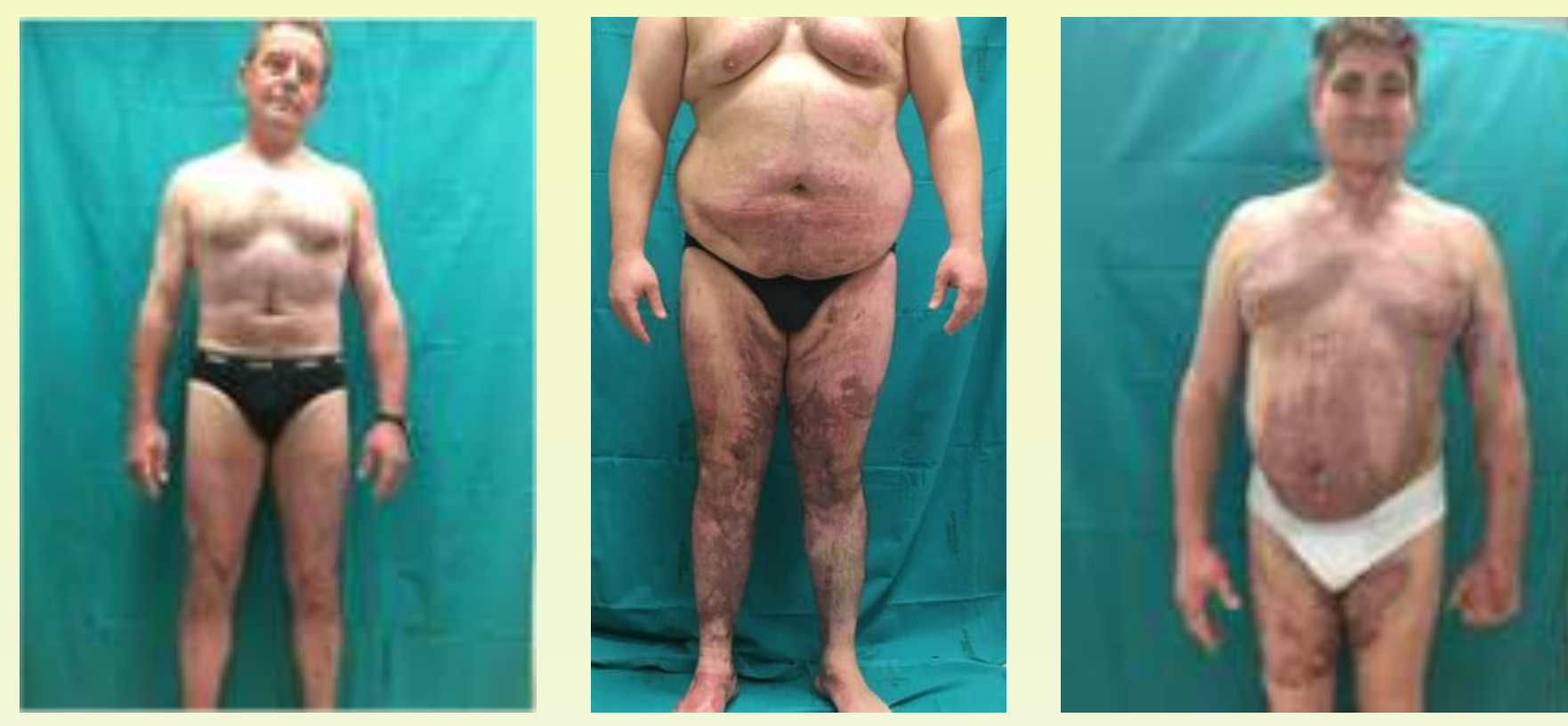

FINAL OUTCOME MOSTLY SCAR FREE EPITHELIALIZED DERMIS

\section{CONCLUSION}

Regardless its a limited number of patient after two years of experience, obtained results are encouraging. Rapid \& selective enzymatic debridement as a minimally invasive tool allowed controlling the burn wound not only on the local level by increasing epithelisation over salvaged dermis and reducing autographing, but it seam also to improve pro and anti-inflammatory response and a better control of SIRS. Further investigations will be necessary to confirm it. 